\title{
Feasible Sanitary Sewer Network Generation Using Graph Theory
}

\author{
Mustafa Erkan Turan $\mathbb{D}^{1},{ }^{1}$ Goksen Bacak-Turan, ${ }^{2}$ Tulin Cetin, ${ }^{1}$ and Ersin Aslan $\mathbb{D}^{3}$ \\ ${ }^{1}$ Manisa Celal Bayar University, Civil Engineering Department, Manisa, Turkey \\ ${ }^{2}$ Manisa Celal Bayar University, Mathematics Department, Manisa, Turkey \\ ${ }^{3}$ Manisa Celal Bayar University, Software Engineering Department, Manisa, Turkey \\ Correspondence should be addressed to Mustafa Erkan Turan; mustafaerkan.turan@cbu.edu.tr
}

Received 19 February 2019; Accepted 7 May 2019; Published 29 May 2019

Academic Editor: Gürol Yildirim

Copyright (C) 2019 Mustafa Erkan Turan et al. This is an open access article distributed under the Creative Commons Attribution License, which permits unrestricted use, distribution, and reproduction in any medium, provided the original work is properly cited.

\begin{abstract}
A graph theory-based methodology is proposed for the sewer system optimization problem in this study. Sewer system optimization includes two subproblems: layout optimization and hydraulic design optimization, which can be solved independently or solved simultaneously. No matter which method is chosen for the solution of the optimization problem, a feasible layout that satisfies the restrictions of the sewer system must be obtained in any step of the solution. There are two different layout options encountered: the layouts containing all sewer links and the layouts not containing all sewer links. The method proposed in this study generates a feasible sewer layout that contains all sewer links and satisfies all restrictions of a sanitary sewer system by using graph theory without any additional strategies unlike other studies. The method is applied to two different case studies. The results of the case studies have shown that graph theory is well applicable to sewer system optimization and the methodology proposed based on it is capable of generating a feasible layout. This study is expected to stimulate the use of graph theory on similar studies.
\end{abstract}

\section{Introduction}

A sewer system, one of the most important structures in urban areas, is built to protect human and environment from the harmful effects of wastewater. Sewer systems consist of pipes, manholes, pumping stations, and other complementary units in general. They can be divided into three types according to the water carried: combined sewer system, storm sewer system, and sanitary sewer system [1]. While storm sewers drain stormwater, sanitary sewers carry sewage and combined sewers carry both.

Sewer system design can be divided into two subproblems. The first is the generation of sewer layout, and the second is the hydraulic design of the predefined layout. The generation of sewer layout problem is to determine the location of the manholes, select the pipes involved, and determine the direction of the flows, whereas the hydraulic design problem is to assess the diameters and slopes of the pipes (e.g., the depth of excavation) and the location of the pump stations [2]. It is tried to avoid using pumping stations and pressurized sewers as much as possible to design according to the gravity flow.

When a suitable sewer network design is made, project cost cannot be ignored. The cost of a project is one of the important criteria for the feasibility of each engineering structure. For this reason, projects with low cost come into prominence within the alternative projects. The cost of a sewer system includes pipe, manhole, excavation, pumping station, wastewater treatment plant, and operating and maintenance costs. Engineering experiences are often used to obtain a low-cost project in the sewer system projects although it is subjective and time-consuming and does not guarantee the optimal cost value. Optimization methods are used to remove this disadvantage and able to introduce more efficiency.

Previous studies on the sewer network optimization problem can be divided into three groups by considering different combinations of the two subproblems mentioned: (1) to find the optimum layout (the first subproblem), (2) simultaneous layout optimization and hydraulic design 
optimization (the first and the second subproblems), and (3) to find the optimum hydraulic design (the second subproblem) [3]. As the independent optimization of two subproblems of the sewer system design may not reach to the global optimum [4] two subproblems must be solved simultaneously to find the global optimum. Despite this, there are more studies on the optimization of the two subproblems. Since the determination of the optimal general layout is a combinatorial optimization problem that is nondeterministic polynomial time complete, it is quite difficult to solve the simultaneous optimization problem $[2,5]$.

Hydraulic design optimization subproblem for the predefined layout is solved by using deterministic methods [6-9] and metaheuristic methods [10-14]. Moreover, different methods are used including both the layout and the hydraulic optimization problems [2,15] as follows. (1) Full enumeration method: having generated all feasible layouts from a base layout, hydraulic design of the layouts obtained is optimized. Finally, a layout with minimum cost is selected as optimum sewer network. In such methods, more than one feasible layout should be generated $[6,16]$. (2) Simplified optimization method: two subproblems are solved independently. At first, the feasible layout is generated and then hydraulic design of obtained layout is optimized [11, 17, 18]. (3) Simultaneous design (or coupled) method: two subproblems are solved simultaneously by using iterative methods [7] or metaheuristic methods $[3,4,19,20]$.

The methods previously mentioned require a feasible layout at any stage of the procedure. Therefore, feasible layout generating methods are needed. A feasible layout of the sewer network must satisfy some restrictions as follows. Since the sewer network system has gravity flow, a sewer system is not allowed to contain loops and a manhole has only one outlet while it can have more than one inlet. Sewer network system layout must be compatible with street layout. All possible manholes should be included in the sewer system layout. Furthermore, all possible sewer links should also be included in a sanitary sewer layout. Finally, the layout must have at least an outlet $[2,4]$.

The methods that generate feasible layouts considering the restrictions above have been developed by different researchers $[2,6,20]$. These methods can be grouped as follows: (1) the methods that generate layouts not including all possible sewer links, which usually generate storm sewer system layouts which include all possible manholes but not all possible sewer links. Therefore, by deleting some of the sewer links, loop-free restrictions can be provided easily. (2) The methods that generate layouts including all possible sewer links, which usually generate sanitary sewer system layouts which include all possible manholes and all possible sewer links. In these methods, the restriction of not to contain loops can be provided by cutting the sewer links from the manholes which constitute loops in the layout. For this purpose, different strategies have been developed.

All possible layouts are obtained by using the connection alternatives of sewer links in the enumeration model in the study of Diogo and Graveto [6]. In this method, there are four connection alternatives for a sewer link between two manholes, say $a$ and $b$, such that the flow direction can be from $a$ to $b$ or from $b$ to $a$, the link can be cut from the manhole $a$ or from the manhole $b$.

The method called loop-by-loop cutting algorithm is developed by Haghighi [2]. This method needs two parameters in order to determine which sewer link to be cut in each loop and from which manhole they are to be cut. Different layouts are obtained according to the parameter values. It is stated that this can be done by trial and error or by an optimization algorithm for which a genetic algorithm is used.

Moeini and Afshar [20] introduce more than one strategy for the purpose of obtaining a layout containing all the sewer links without loops. In the first strategy, the pipes are cut at the upstream end of the manholes. In the second strategy, the pipes are cut at the manholes with the highest rank where the rank is the minimum number of the pipes between the manhole and the outlet. In the third strategy, the location of the cut end is determined as the pipe with the steeper slope and the cut end with a minimum allowable cover depth.

In the studies mentioned above, an initial layout called base layout and including all possible manholes and sewer links according to the street layout is obtained. This network does not satisfy the restrictions since the base layout obtained contains loops. Therefore, the sewer links are determined to cut in each loop and cut from any end according to the chosen strategy in order to obtain a feasible layout that satisfies the restrictions. The strategies proposed differ from researcher to researcher. In addition, these strategies may require the use of several methods together.

Graph theory is one of the most preferred methods by the researchers when considering the used methods in the sewer system optimization problem [2, 4, 5, 7, 17, 20-25]. The use of graph theory in sewer optimization provides several advantages. Graph representation of sewer networks is computationally very efficient [5] enabling the application of graph theory concepts and theorems on graphs. In some studies, the layout is modelled by using a graph only for representation and the methods developed according to this representation are applied [2, 5]. In most applications, shortest-path and minimum spanning tree algorithms are used. The purpose of such studies is to obtain the shortest path and/or the lowest cost network in one step $[7,16,17,26]$.

In this study, a method is proposed that generates a feasible sanitary sewer layout which satisfies all the restrictions above completely by using graph theory concepts and methods without any additional strategies. In most of the methods, the researchers used more than one strategy to obtain the sewer layout that includes all the sewer links. The proposed method is more understandable, easy to apply, and efficient because it contains the known concepts of graph theory. In this study, to show the applicability of the method, two different applications are given including previous studies in the literature, generating shortest-path spanning tree layout and generating more than one feasible layout. 


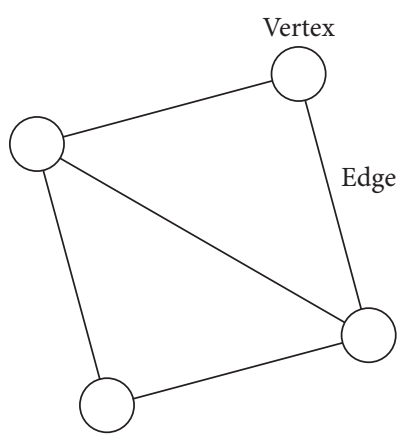

(a)

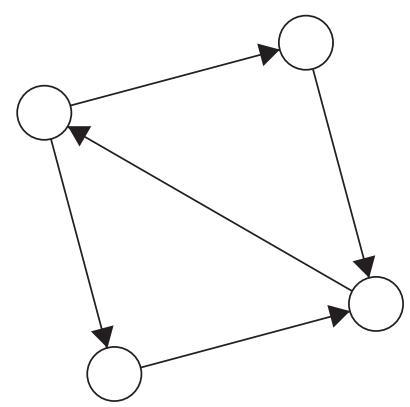

(b)

Figure 1: (a) Undirected graph. (b) Directed graph.

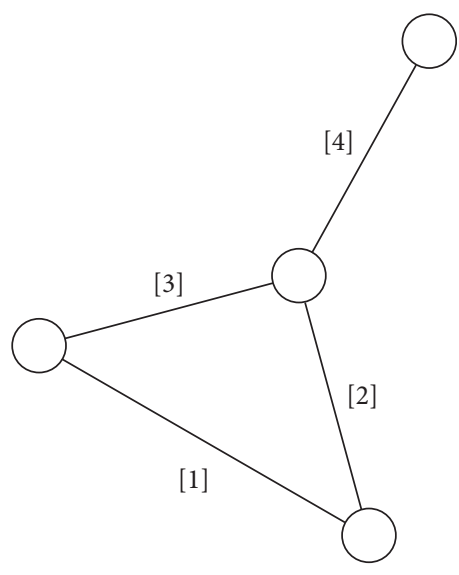

(a)

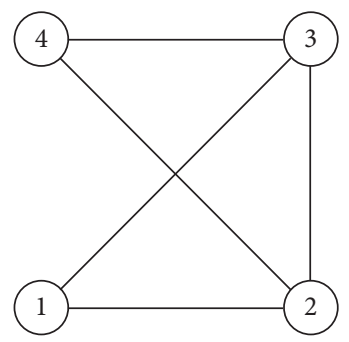

Figure 2: (a) Graph $G$. (b) Line graph $L(G)$ of $G$ [27].

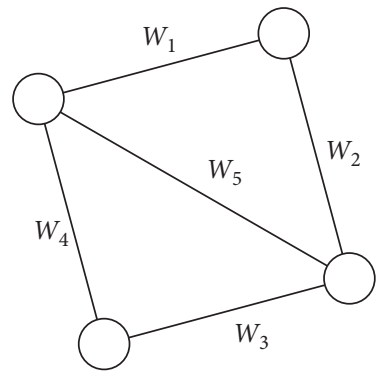

(a)

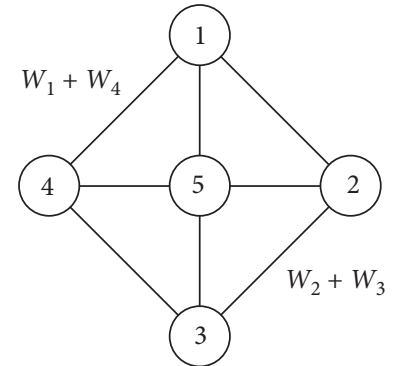

(b)

Figure 3: (a) Weighted base graph. (b) Weighted line graph.

\section{Graph Theory and Sewer Network Representation}

Graph theory is a branch of mathematics which helps solving problems encountered in daily life more easily by modelling graphs. A graph is a structure in which a situation or an object is represented by a node and the related situations or objects are connected by a line. Structures called networks usually correspond to a graph.
A graph can be represented by $G=(V, E)$ where $V(G)$ is the set of vertices (nodes) and $E(G)$ is the set of edges (links). An edge can be denoted by the ordered pair $(u, v)$ where $u$ and $v$ are the end vertices of the edge. Adjacency matrix and incidence matrix are the matrix forms for the representation of graphs. The adjacency matrix has the rows and columns labeled by the labels of the vertices. If an entry is different from zero, the labeled vertices corresponding to that row and that column have an edge connecting them. The incidence 


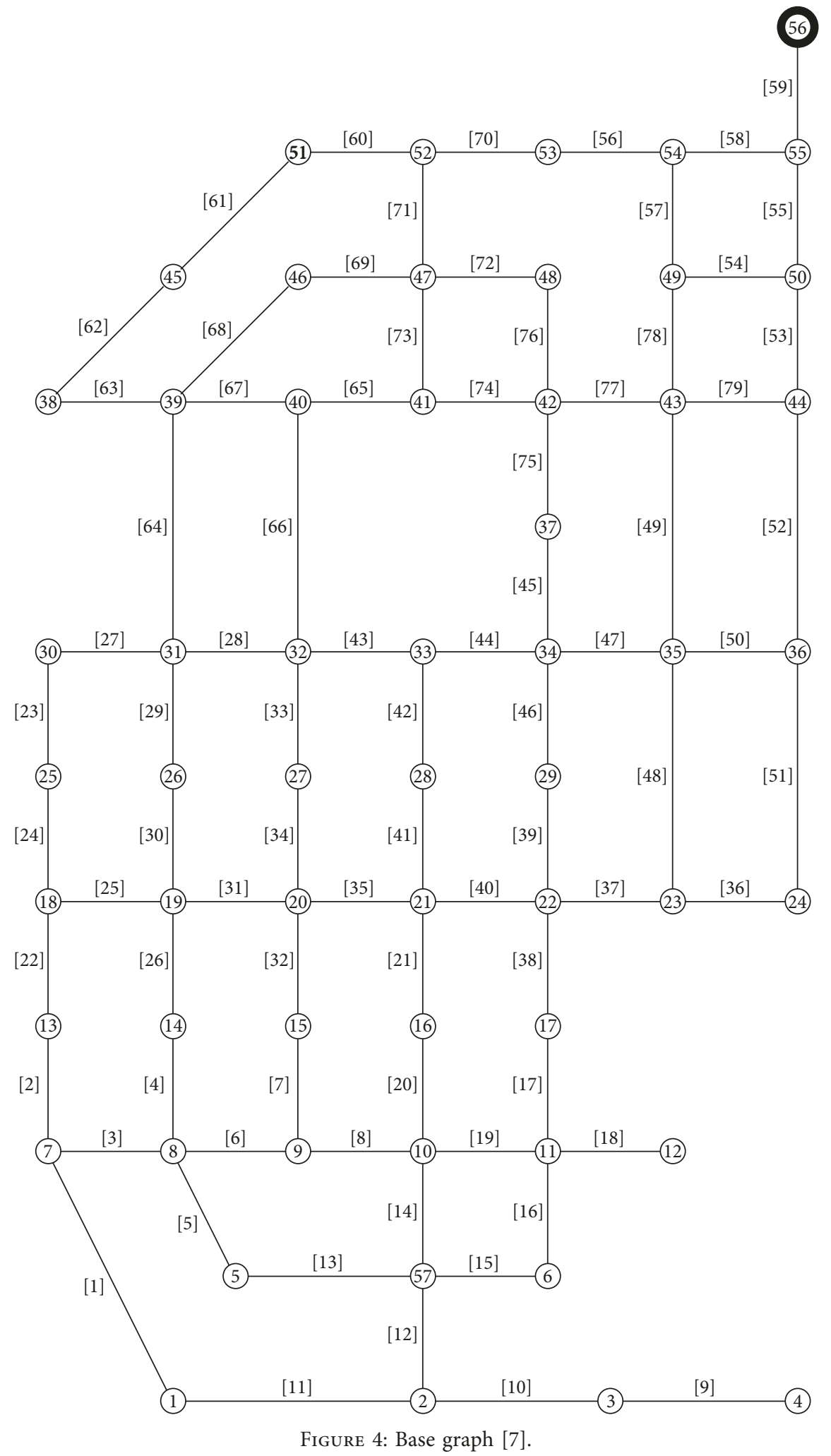

matrix has the rows and columns labeled by the vertices and edges, respectively. If an entry is different from zero, the labeled vertex corresponding to that row is an end vertex of the labeled edge corresponding to that column. The graphs are called directed graphs if the edges are directed, i.e., a directed $(u, v)$ edge has an arrow from vertex $u$ to vertex $v$ given in Figure 1(b), otherwise called undirected graphs given in Figure 1(a). If the edges of a graph are associated with some numerical values, the graph is called a weighted graph. A graph is called a connected graph when there is a 


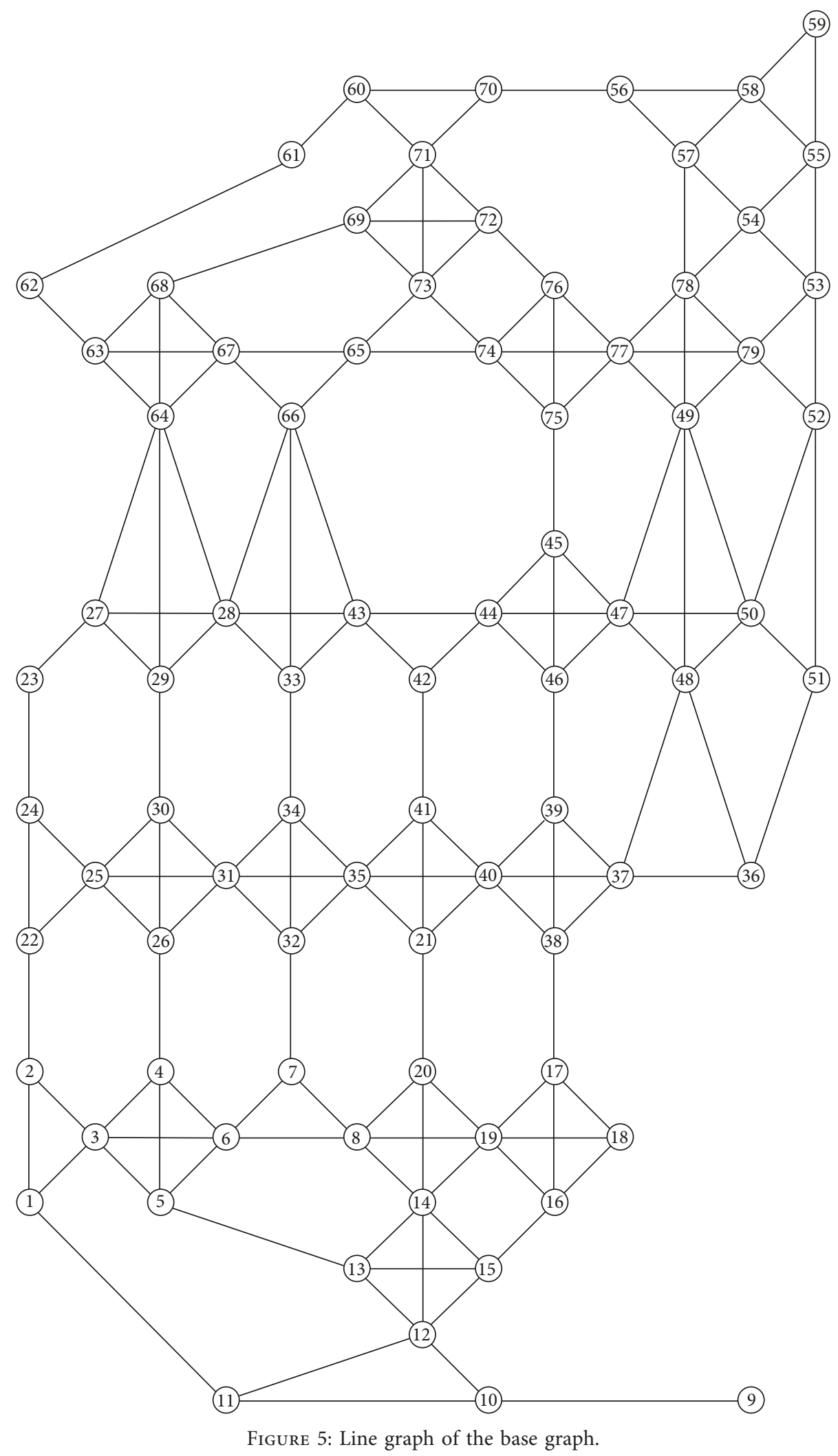

path between every pair of vertices, otherwise called a disconnected graph. A loop (cycle) is a path that begins and ends at the same vertex. Graphs without loops are called trees. A spanning tree of a graph is a subgraph that is a tree containing all the vertices of the graph. Minimum spanning tree has the minimum total edge weight among the spanning trees of a graph. A shortest-path tree for a graph $G$ from a vertex $v$ (root) is a rooted tree containing all the vertices of $G$ 


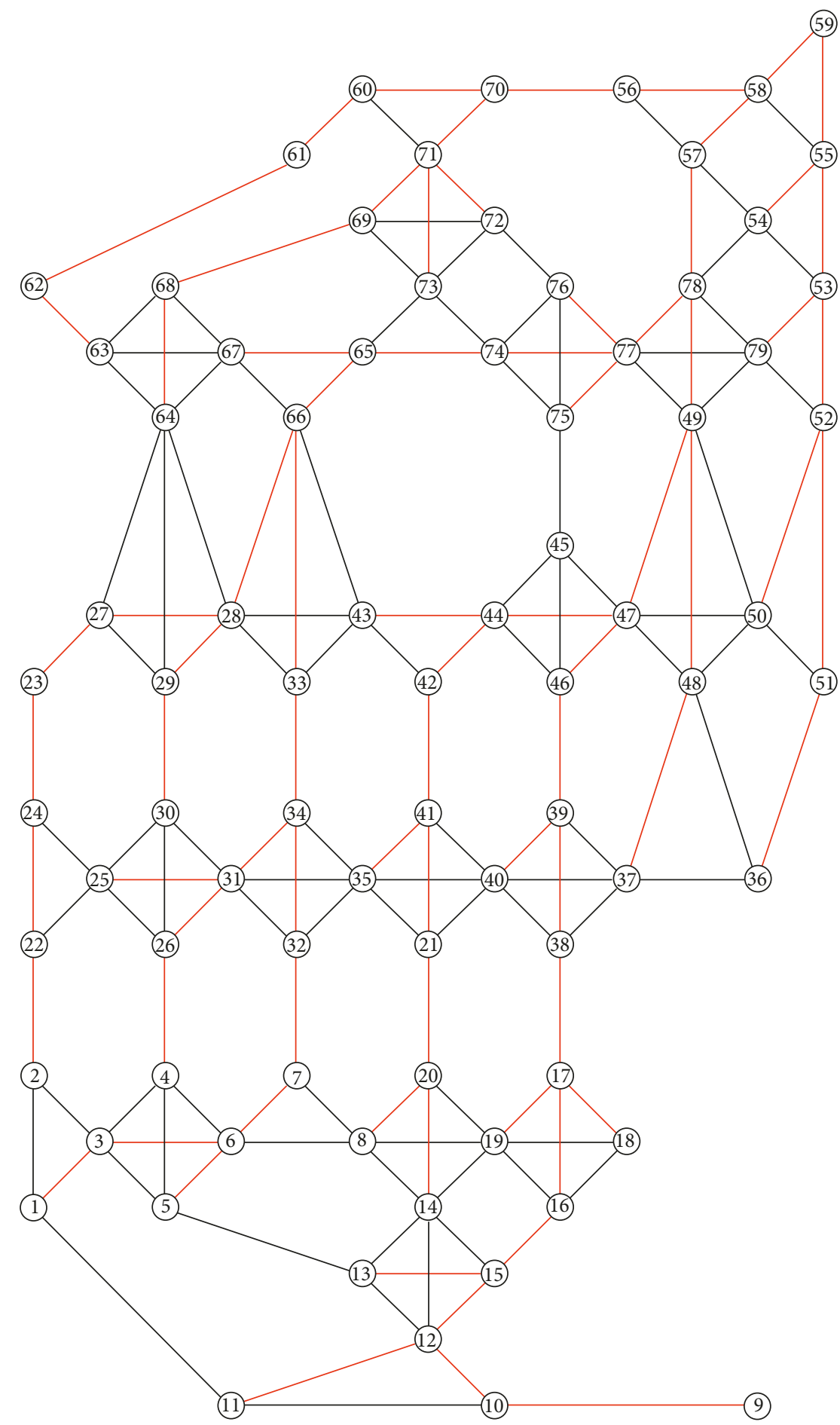

FIgURE 6: Shortest-path spanning tree of the line graph.

such that the unique path in the tree from $v$ to each vertex $w$ in $G$ is a shortest path in $G$ from $v$ to $w$ [27].

The line graph $L(G)$ given in Figure 2(b) of a graph $G$ given in Figure 2(a) has a vertex for each edge of $G$, and two vertices in $L(G)$ are adjacent if and only if the corresponding edges in $G$ have a vertex in common [27].
A sewer network can be modelled by a graph representing manholes and sewer links by vertices and edges, respectively. A graph that consists of all possible vertices (manholes) and edges (sewer links) called a base graph as it is called a base layout previously. In this form, a base graph contains loops and undirected edges. The restrictions given 


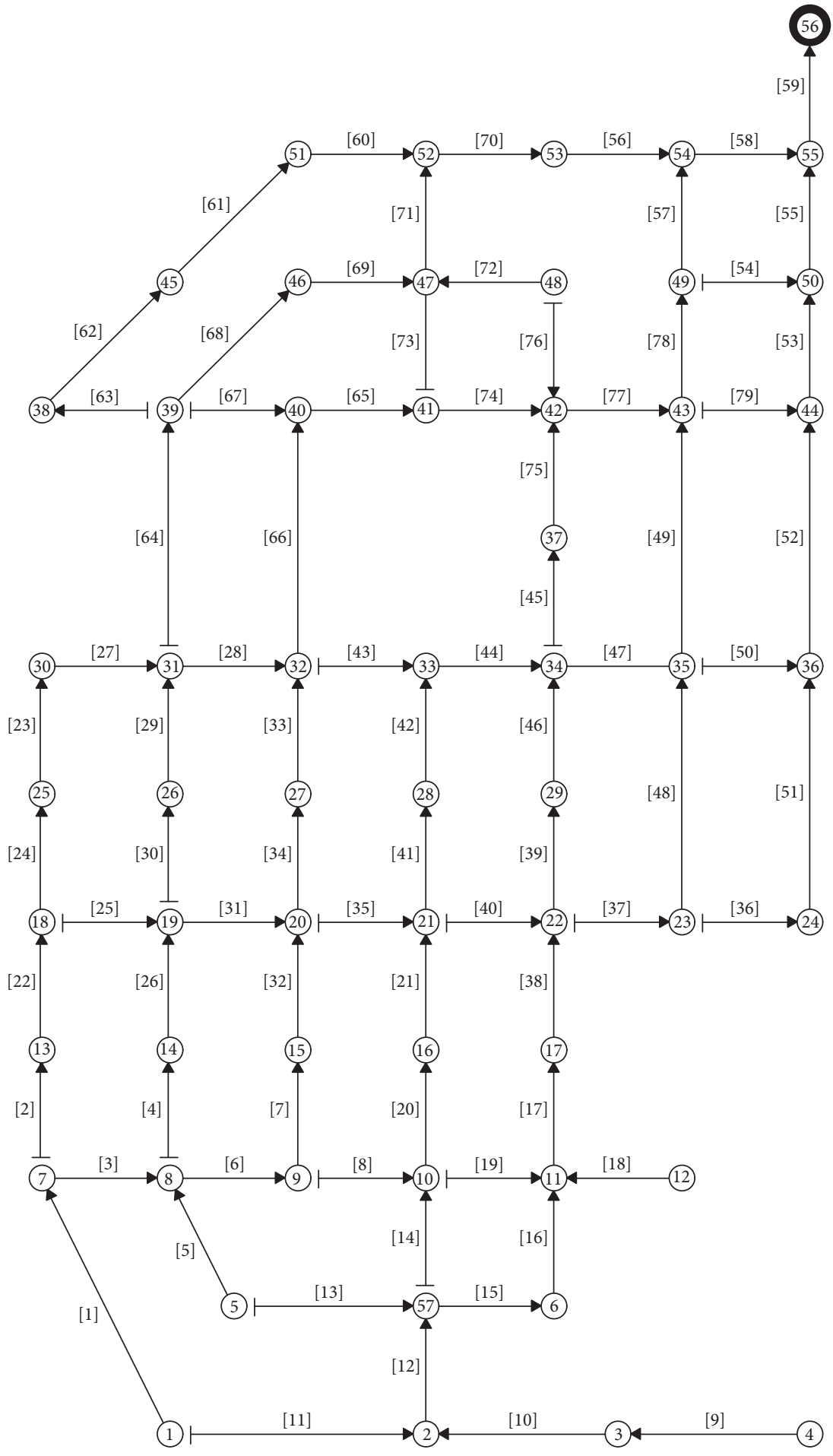

FIgURE 7: Consequence layout.

for a sanitary sewer system previously are explained below corresponding to the graph theory terminology:

(1) A sewer system is not allowed to contain loops. In graph theory, this corresponds that the graph is a tree.
(2) A sanitary sewer system should contain all manholes (vertices). In graph theory, this corresponds that the tree is a spanning tree.

(3) A sanitary sewer system should contain all sewer links. The graph should contain all the edges. 


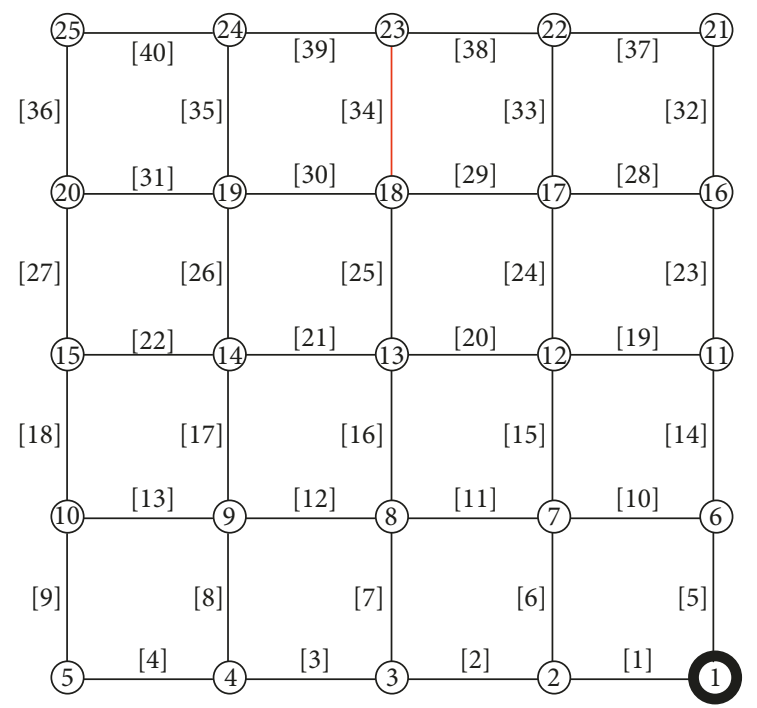

(a)

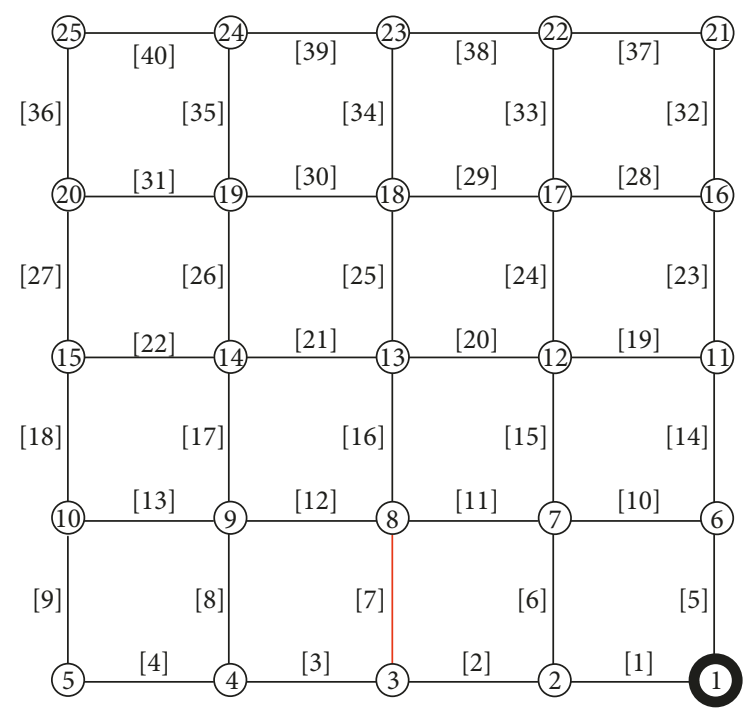

(c)

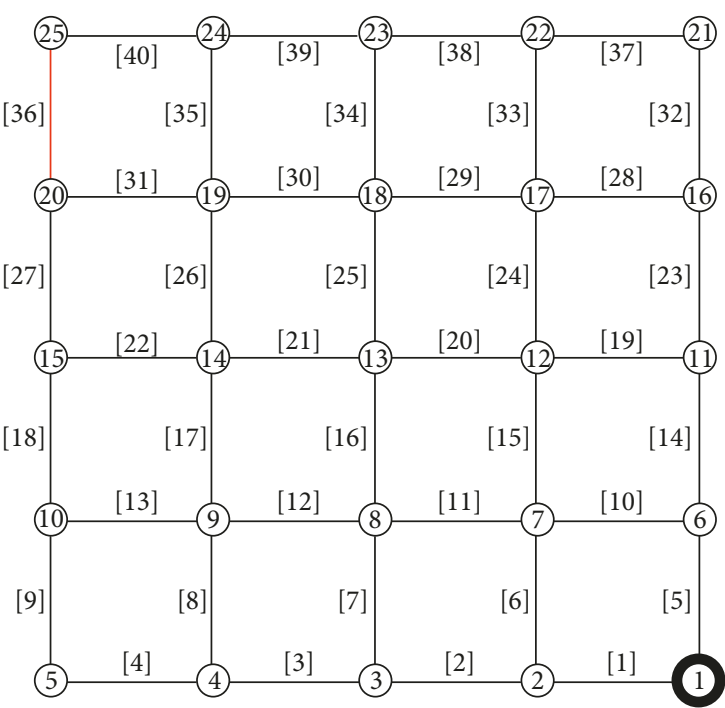

(b)

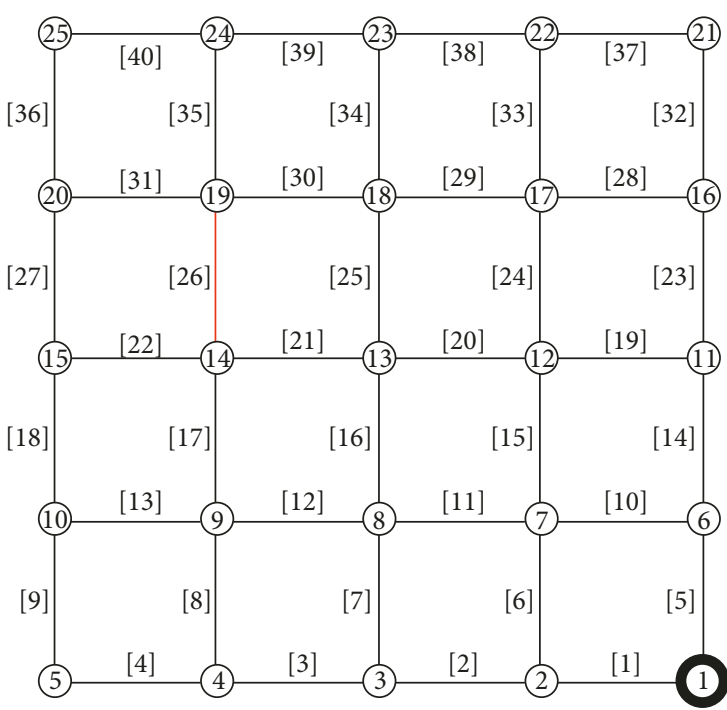

(d)

Figure 8: Base graphs.

(4) A manhole can have more than one inlet but only one outlet. In graph theory, this corresponds that the outdegree of each vertex is one and the indegree can be greater than one excluding target.

(5) Spanning tree of the sewer network should be directed to a target [2].

\section{The Method Proposed}

The proposed method generates a sewer system network layout from a base layout in such a way that it contains all possible sewer links and it satisfies all the restrictions of the sewer systems mentioned previously. Unlike the methods used for the same purpose, graph theory concepts and methods are used only with no additional strategies to satisfy the restrictions of sewer network layout.
A tree based on its definition is obtained containing all the vertices but not containing all the edges in the algorithms, such as the minimum spanning tree and the shortestpath tree algorithms, as a result of which the spanning tree is obtained. In the proposed method, the base graph is transformed into its line graph to include all the edges before applying the algorithms, while the additional strategies are used to include all the edges in many studies. The key point is to obtain the line graph of the base graph by modelling each edge in the base graph as a vertex in the line graph to transform a base graph into a line graph. Thus, a tree is obtained with all the vertices corresponding to the all edges of the base graph when an algorithm that produces a spanning tree is applied to this line graph. As a result, when the line graph is back transformed into the base graph, a tree is obtained containing all the edges of the base graph. The proposed method consists of four steps as follows: 
Step 1. Obtaining base graph.

In the first step, the base graph $G$ that is a connected and undirected graph containing all the possible edges (sewer links), all the possible vertices (manholes), and the target (outlet) is constructed according to the street layout. The incidence matrix $M$ is used to represent the base graph obtained since it is necessary in the next step.

Step 2. Obtaining line graph.

The adjacency matrix of the line graph of the base graph $A_{\mathrm{L}}$ is obtained by using the incidence matrix of the base graph $G$ according to the following equation [28]:

$$
A_{\mathrm{L}}=M M^{\mathrm{T}}-2 I \text {, }
$$

where $A_{\mathrm{L}}$ represents the adjacency matrix of the line graph, $M$ represents the incidence matrix of the base graph, $M^{\mathrm{T}}$ represents the transpose of the matrix $M$, and $I$ represents the identity matrix.

\section{Step 3. Obtaining spanning tree.}

In this step, a graph algorithm is applied to the line graph to obtain a spanning tree. There are several graph algorithms to generate a spanning tree for the weighted or unweighted graphs. Determination of a suitable algorithm depends on the purpose of the study to be performed.

If the algorithm used for generating spanning trees needs weighted graphs, the line graph should be a weighted graph. This also requires the transfer of weights to the line graph during the transformation of the base graph into the line graph. When the base graph is transformed into the line graph, the weights of the base graph will be assigned to the vertices of the line graph since the edges of the base graph corresponds to the vertices of the line graph. Therefore, the weights should be assigned to the edges. The corresponding edges to those vertices have a common end vertex in the base graph when two vertices are connected by an edge in the line graph. Hence, the weight of an edge in the line graph is the sum of the weights of the two edges in the base graph given in Figure 3(a) corresponding to the end vertices of this edge and given in Figure 3(b).

\section{Step 4. Obtaining consequence graph.}

Since the spanning tree obtained belongs to the line graph, it should be back transformed into the base graph. After the back transformation, the corresponding edges in the base graphs to the vertices of the spanning trees in the line graph constitute the spanning trees including all the edges with their directions to the outlet. If two edges have a common end vertex in the base graph but the corresponding vertices in the spanning tree of the line graph are not connected, one of the edges is cut according to their directions and connections to the other edges. Hence, the spanning trees including all the edges of the base graphs are formed. As a result, a feasible layout that satisfies all the restrictions of a sewer system is obtained.

\section{Application and Results}

In this section, in order to show the applicability of the method proposed in the previous section, two different applications: generating shortest-path spanning tree layout and generating more than one feasible layout, are given based on the previous studies in the literature.

4.1. Generating Shortest-Path Spanning Tree Layout. Tekeli and Belkaya [17] obtained a sewer system layout by using shortest-path spanning tree and minimum spanning tree algorithms. In this method, a layout containing all the edges is not obtained. Li and Matthew [7] suggested a searching direction iterative method and obtained a shortestpath spanning tree by using Dijkstra's algorithm at the first step. As for the above studies, when a minimum spanning tree or shortest-path spanning tree algorithm is applied to the base graph, a graph including all the edges cannot be obtained. On the contrary, when the steps of the proposed method are applied, a layout that includes all the edges is obtained using the same algorithms. Turan et al., [26] made a preliminary application of the proposed method on an unweighted graph.

The sewer system layout introduced by Li and Matthew [7] is used for the case study of this section. The sewer network has an outlet with 56 manholes and 79 sewer links with the $56^{\text {th }}$ manhole representing the outlet. Layout specifications can be found in $\mathrm{Li}$ and Matthew [7]. The results of the case study are given in detail as follows:

\section{Step 1. Obtaining base graph.}

The base graph considered is given in Figure 4, and the corresponding incidence matrix of the base graph is given in the following equation:

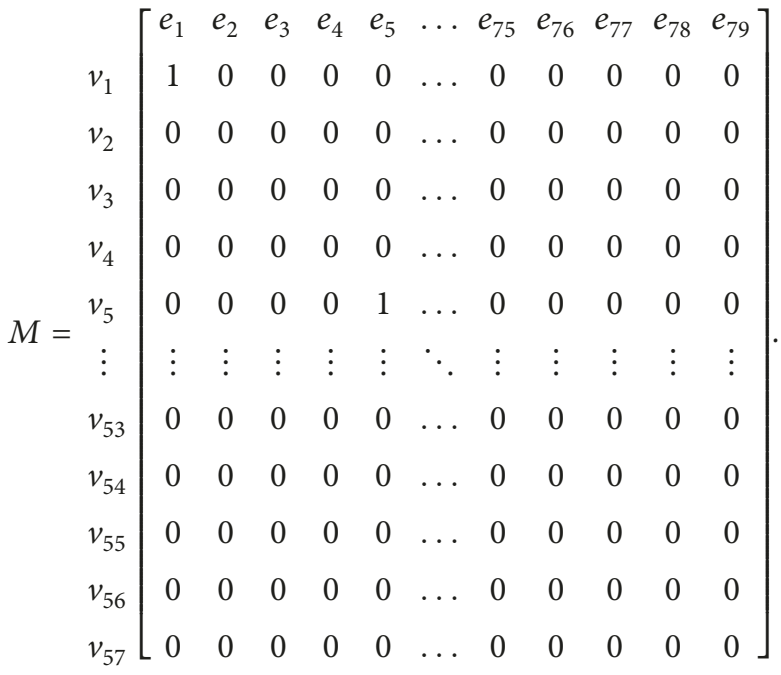

Step 2. Obtaining line graph.

The line graph of the base graph is obtained by using equation (1). The adjacency matrix and the line graph are given in the following equation and Figure 5, respectively: 


$$
A_{\mathrm{L}}=\begin{gathered}
v_{1} \\
v_{2} \\
v_{3} \\
v_{4} \\
\vdots \\
v_{75} \\
v_{76} \\
v_{77} \\
v_{78} \\
v_{79}
\end{gathered}\left[\begin{array}{ccccccccccc}
v_{1} & v_{2} & v_{3} & v_{4} & v_{5} & \ldots & v_{75} & v_{76} & v_{77} & v_{78} & v_{79} \\
0 & 1 & 1 & 0 & 0 & \ldots & 0 & 0 & 0 & 0 & 0 \\
1 & 0 & 1 & 0 & 0 & \ldots & 0 & 0 & 0 & 0 & 0 \\
1 & 1 & 0 & 1 & 1 & \ldots & 0 & 0 & 0 & 0 & 0 \\
0 & 0 & 1 & 0 & 1 & \ldots & 0 & 0 & 0 & 0 & 0 \\
0 & 0 & 1 & 1 & 0 & \ldots & 0 & 0 & 0 & 0 & 0 \\
\vdots & \vdots & \vdots & \vdots & \vdots & \ddots & \vdots & \vdots & \vdots & \vdots & \vdots \\
0 & 0 & 0 & 0 & 0 & \ldots & 0 & 1 & 1 & 0 & 0 \\
0 & 0 & 0 & 0 & 0 & \ldots & 1 & 0 & 1 & 0 & 0 \\
0 & 0 & 0 & 0 & 0 & \ldots & 1 & 1 & 0 & 1 & 1 \\
0 & 0 & 0 & 0 & 0 & \ldots & 0 & 0 & 1 & 0 & 1 \\
& 0 & 0 & \ldots & 0 & 0 & 1 & 1 & 0
\end{array}\right] .
$$

\section{Step 3. Obtaining spanning tree.}

Dijkstra's algorithm, which is one of the shortest-path spanning tree algorithms, is used as in Li and Matthew [7] to compare the results. The details of this algorithm can be found in the study of Dijkstra [29]. A weighted graph is needed to run this algorithm. The cost function introduced in Li and Matthew [7] given in the following equation is used to calculate the weights:

$$
C=\sum_{i=1}^{n} L_{i} \bar{E}_{i}
$$

where $C$ is the cost, $L_{i}$ is the length of the pipe, and $\bar{E}_{i}$ is the average ground elevation.

The adjacency matrix of the weighted line graph is given in the following equation, and the shortest-path spanning tree of the line graph represented by red colored edges is given in Figure 6:

$$
A_{\mathrm{L}}=\begin{gathered}
v_{1} \\
v_{2} \\
v_{3} \\
v_{4} \\
v_{5} \\
\vdots \\
v_{75} \\
v_{76} \\
v_{77} \\
v_{78} \\
v_{79}
\end{gathered}\left[\begin{array}{ccccccccccc}
v_{1} & v_{2} & v_{3} & v_{4} & v_{5} & \ldots & v_{75} & v_{76} & v_{77} & v_{78} & v_{79} \\
0 & 1660,25 & 1560 & 0 & 0 & \ldots & 0 & 0 & 0 & 0 & 0 \\
1560 & 1492,25 & 0 & 1357,5 & 1359 & \ldots & 0 & 0 & 0 & 0 & 0 \\
0 & 0 & 1357,5 & 0 & 1324,5 & \ldots & 0 & 0 & 0 & 0 & 0 \\
0 & 0 & 1359 & 1324,5 & 0 & \ldots & 0 & 0 & 0 & 0 & 0 \\
0 & 0 & \vdots & \vdots & \vdots & \ddots & \vdots & \vdots & \vdots & \vdots & \vdots \\
0 & 0 & 0 & 0 & 0 & \ldots & 0 & 937,5 & 902 & 0 & 0 \\
0 & 0 & 0 & 0 & 0 & \ldots & 937,5 & 0 & 1224,5 & 0 & 0 \\
0 & 0 & 0 & 0 & \ldots & 902 & 1224,5 & 0 & 1154,5 & 1214,5 \\
0 & 0 & 0 & 0 & \ldots & 0 & 0 & 1154,5 & 0 & 1180 \\
0 & 0 & 0 & 0 & 0 & 0 & 0 & 1214,5 & 1180 & 0
\end{array}\right] .
$$

Step 4. Obtaining consequence graph.

In this step, the consequence graph is obtained by back transformation of the line graph into the base graph and given in Figure 7.

The graph obtained is a feasible layout that satisfies all the requirements of a sewer system. The results are compared with those of Li and Matthew [7]. The consequence layout and the layout of the shortest-path spanning tree in the study of Li and Matthew [7] are exactly the same. These results show that the process steps and the assumptions made are consistent.

4.2. Generating Multiple Layouts. It is envisaged that it is sufficient for the sewer layout produced in some studies to satisfy only the restrictions mentioned previously. Navin et al. [16] produced all spanning trees by applying Kruskal's algorithm after removing one edge from the base graph at each step. Then, they chose the optimum layout by sorting them in the ascending order after the calculation of the sum of the cumulative discharges for all the generated layouts. Diogo et al. [5] generated feasible layouts by using the forest algorithm used in the simulating and genetic algorithm methods. Diogo and Graveto [6] obtained all feasible layouts by the enumeration model, and then they obtained the optimum sewer system by hydraulic design optimization of all layouts. Walters and Smith [25] generated a random feasible layout with the tree growing algorithm to genetic algorithm. Similarly, in this study the layouts containing all the edges have not been obtained. As in these studies, more than one feasible layout is necessary to be produced. For this purpose, in some studies removing an edge or adding an edge in the base graphs are used to produce different layouts $[5,16]$. However, removing an edge is not possible in this study since the purpose of the proposed method is to obtain a feasible layout that includes all the edges. Therefore, as in the previous case study, different layouts are obtained by 


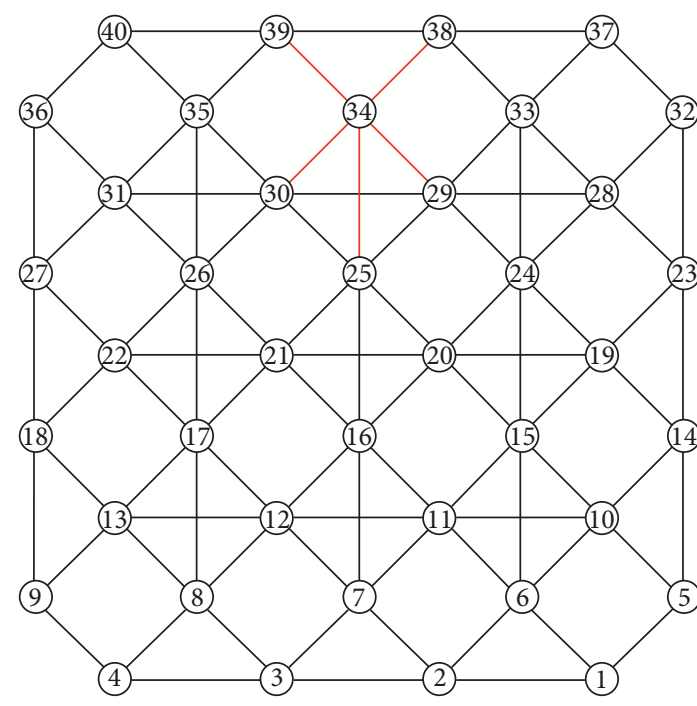

(a)

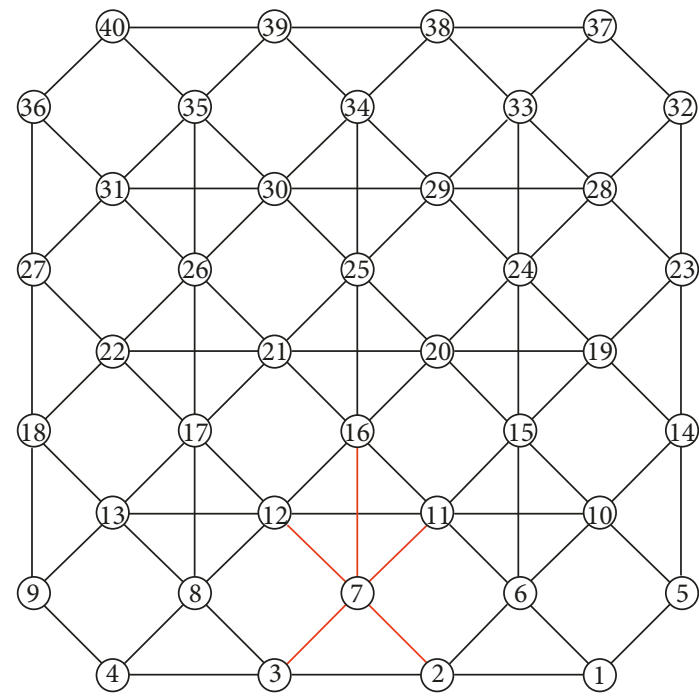

(c)

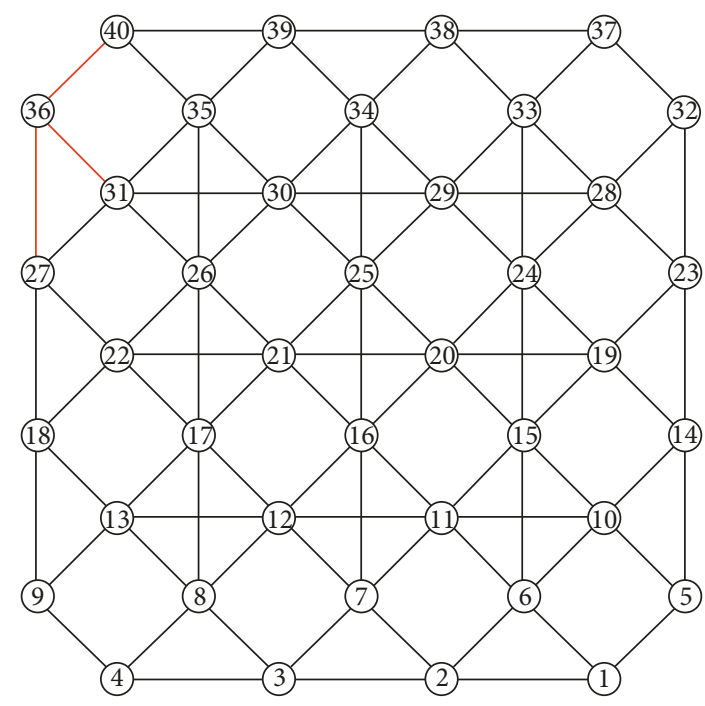

(b)

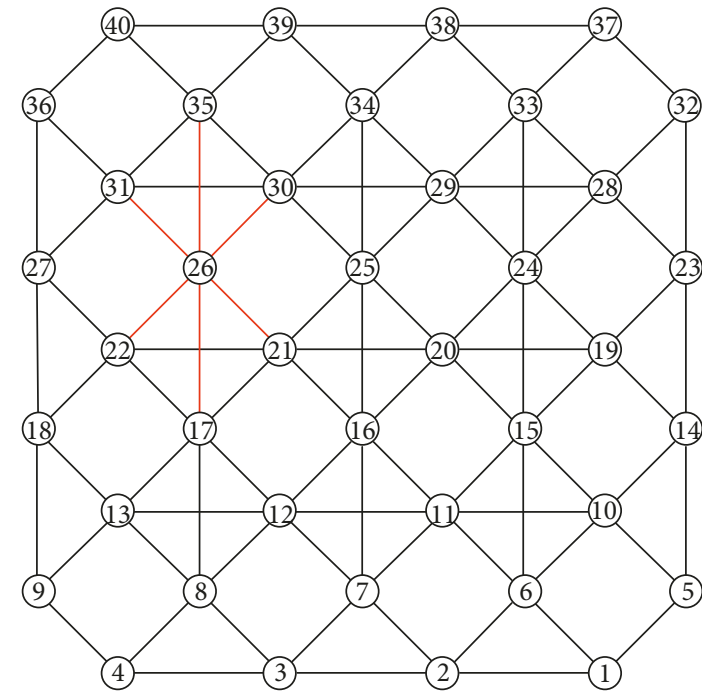

(d)

Figure 9: Line graphs.

reassigning the weights on the edges of the base graph at random when a generating spanning tree algorithm that runs on the weighted graphs is used. For example, a feasible layout is required for each individual when the populationbased metaheuristic optimization methods are used. A feasible layout can be generated for each individual by the proposed method with an additional step. The steps of this version of the proposed method are given below:

(1) Obtain a base graph

(2) Assign a large value as a weight to any of the edges of the base graph randomly

(3) Obtain a line graph

(4) Obtain a spanning tree

(5) Obtain a consequence graph

Except for the second step, the steps above are the same with the steps of the method proposed. The desired number of feasible layouts can be generated by running this version of the method repeatedly.

The hypothetic sewer system that was introduced by Moeini and Afshar [19] is used for the case study of this section. The sewer network has an outlet, 25 manholes, and 40 sewer links with the first manhole representing outlet. Layout specifications can be found in the study of Moeini and Afshar [19].

The generation of four different feasible layouts is aimed for this application. For this purpose, the steps given above have been repeated four times. For the first step, the base graph is taken from the study of Moeini and Afshar [19] as it is. In the second step, an edge is selected randomly from the base graph and a large value is assigned to this edge as weight. For the first repetition, the randomly selected edge labeled as 34 in the base graph is marked red as shown in Figure 8(a). For the remaining repetitions, the randomly selected edges labeled 36, 7, and 26 in the base 


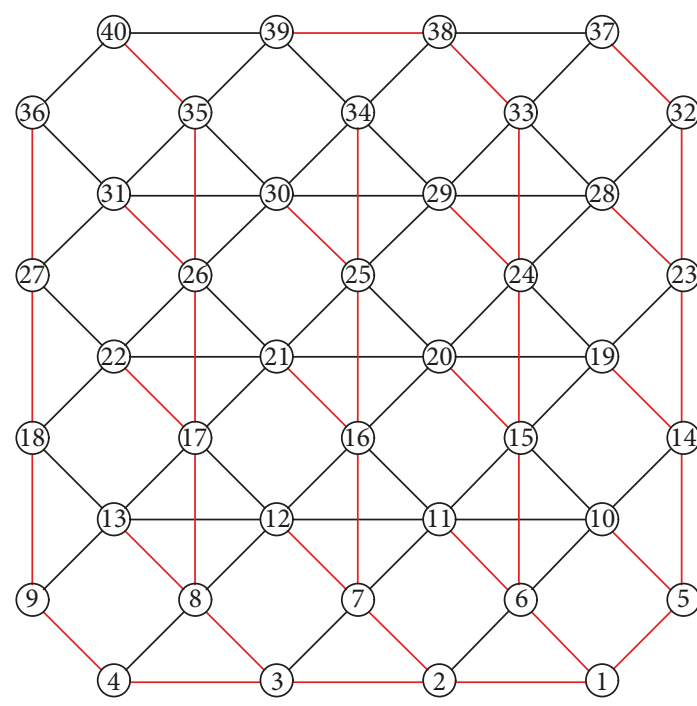

(a)

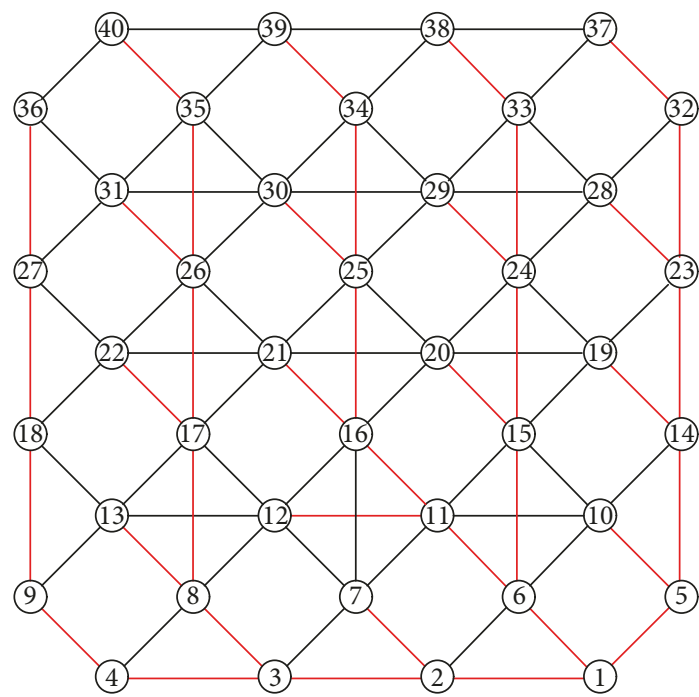

(c)

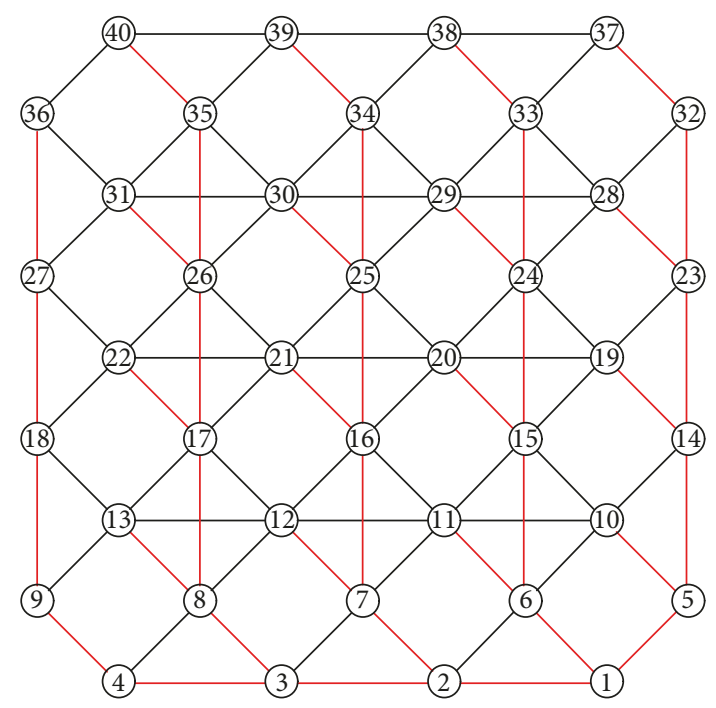

(b)

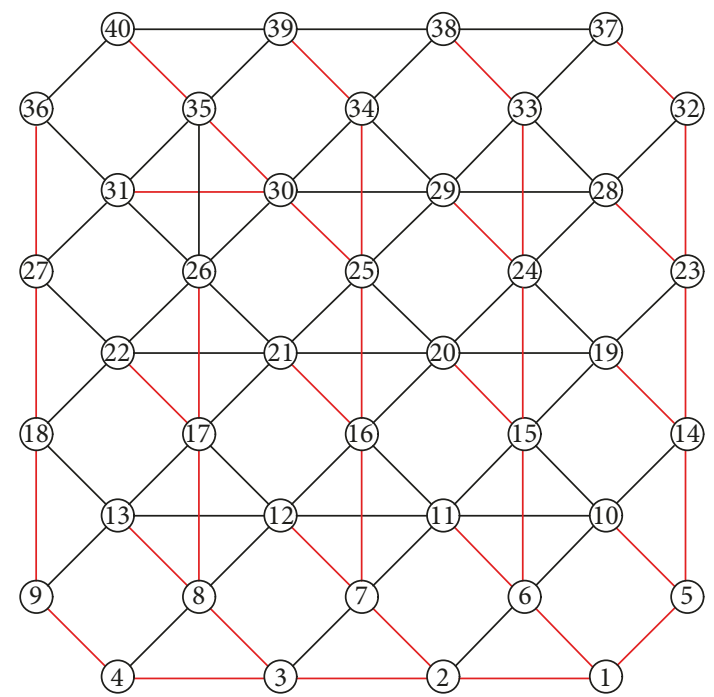

(d)

Figure 10: Shortest paths of the line graphs.

graph are marked red as shown in Figures $8(b)-8(d)$, respectively.

The line graphs obtained in the third step are given for every repetition in Figure 9. Since the base graphs have weighted edges, the corresponding vertices in the line graphs have the corresponding weights. The red edges labeled as 34, 36,7 , and 26 having large values as weights in the base graphs in Figure 8 correspond to the vertices with the same labels in Figure 9, respectively. The edge weights in the line graphs are equal to the sum of the weights of the end vertices of the edges. The weights of these vertices affect all the incident edges. The red colored edges with large weight values are given for every repetition in Figure 9.

In the fourth step, a spanning tree for each line graph is obtained by using Dijkstra's algorithm as in the previous case study. Some of the edges colored red are chosen by using the algorithm to constitute the shortest paths between all the vertices and the target given in Figure 10. The red colored edges are directed to the target constituting the spanning trees given in Figure 11.

In the final step, the four different consequence layouts are obtained by back transformation of the above line graphs into the base graphs. The consequence layout obtained at the end of the first repetition is given in Figure 12(a). Figures 12(b)-12(d) represent the consequence layouts for the remaining repetitions, respectively.

When the results are examined, it is possible to obtain more than one layout that satisfies sewer network restrictions corresponding to the same base graph by this version of the proposed method.

In this case study, it is explained how to generate feasible layouts as the number of individuals in a population-based optimization method. All feasible layouts can be generated by assigning large values of weights to the edges of the base graph in a certain order when all feasible layouts are desired. 


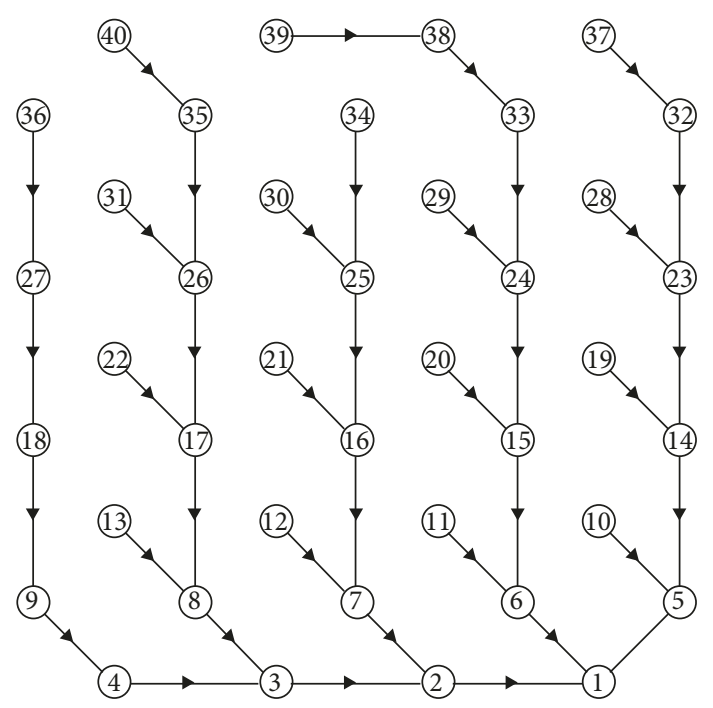

(a)

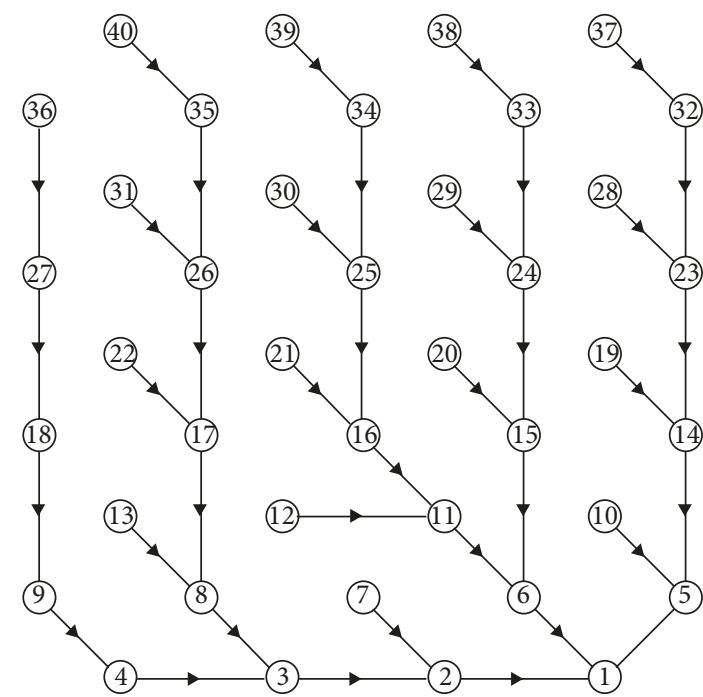

(c)

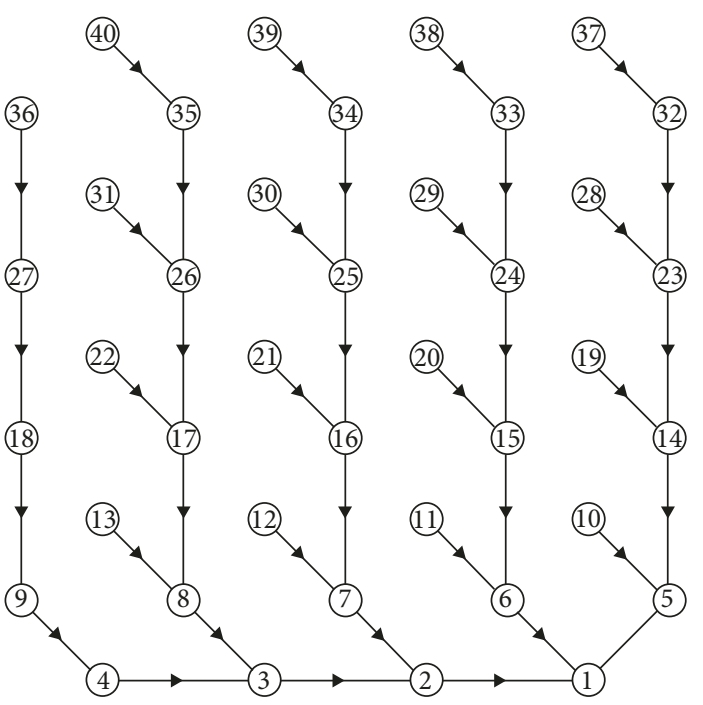

(b)

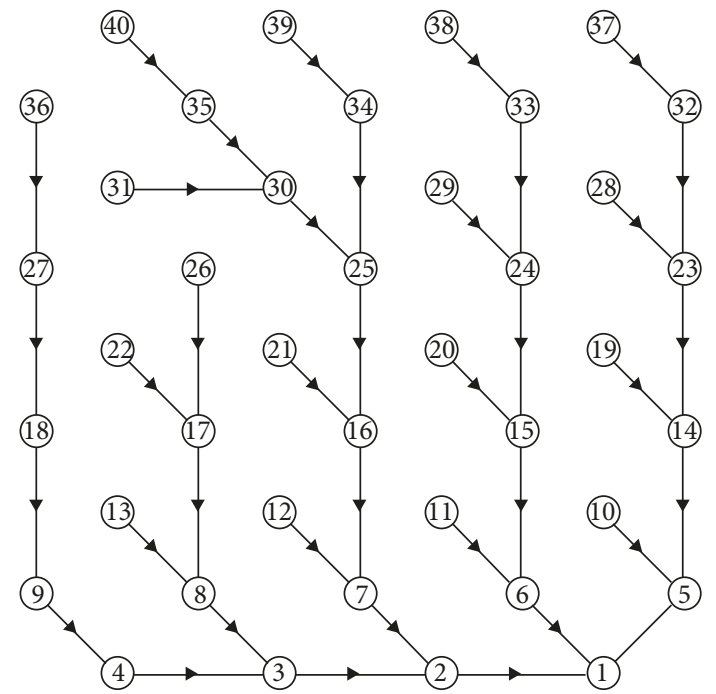

(d)

FIgURE 11: Spanning trees of the line graphs.

\section{Conclusion}

As one of the most important elements of an urban infrastructure, sewer system optimization has been discussed in this study. The optimization problem is divided into two subproblems: layout optimization and hydraulic design optimization. While most of the studies in the literature search for a solution to the hydraulic design optimization problem, the layout optimization seems to take less attention as an optimization problem. However, it is very important to generate a feasible layout prior to performing hydraulic design. Two different types of sewer system layouts can be generated for different purposes. One of them is to obtain a layout that contains all possible manholes, and the other is to obtain a layout with all possible manholes together with all sewer links. Different methods have been developed by different researchers to obtain a layout that contains all sewer links. These methods often involve more than one strategy/method.

In this study, unlike the previously proposed methods, a method which does not require an additional strategy based on graph theory concepts and methods has been proposed. Therefore, the proposed method is more understandable, easy to apply, and efficient because it contains the known concepts of graph theory. The simplest form of the method proposed consists of four steps. In the first step, a base graph is obtained according to the street layout. The second step, which is the most important step of the study, is to obtain the line graph from the base graph. In the third step, a spanning tree algorithm is applied to the line graph. In the last step, a consequence layout satisfying all the restrictions and containing all sewer links is obtained by back transformation. The method proposed in this study can be used in the process of obtaining a feasible layout in the methods 


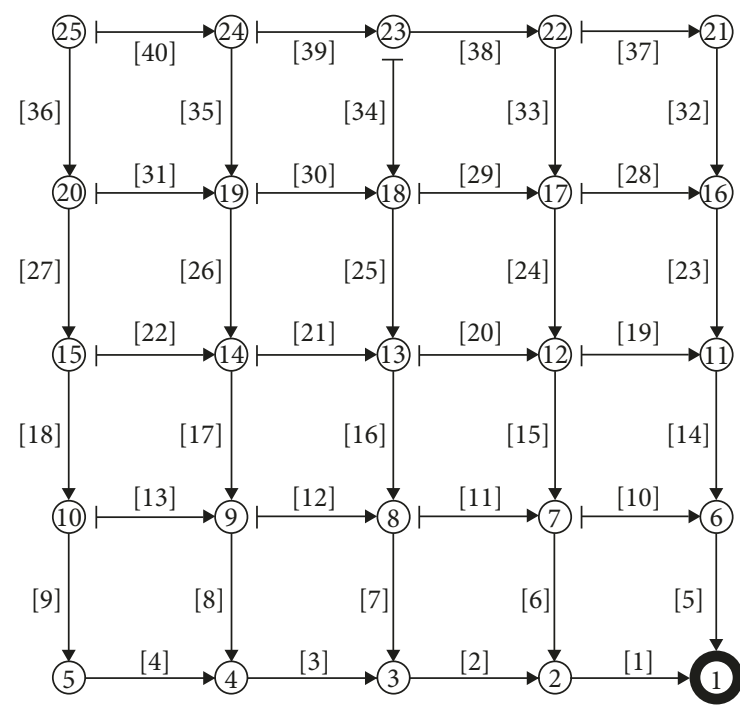

(a)

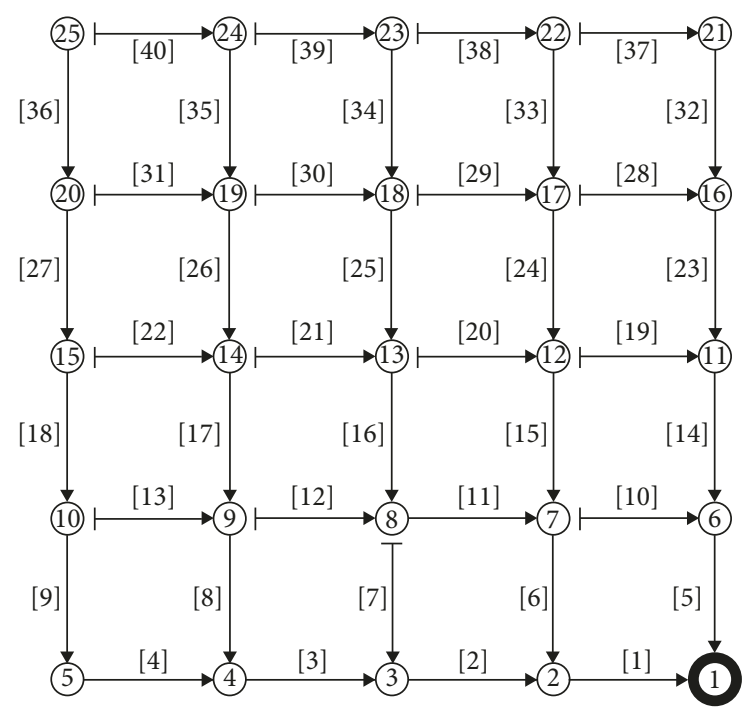

(c)

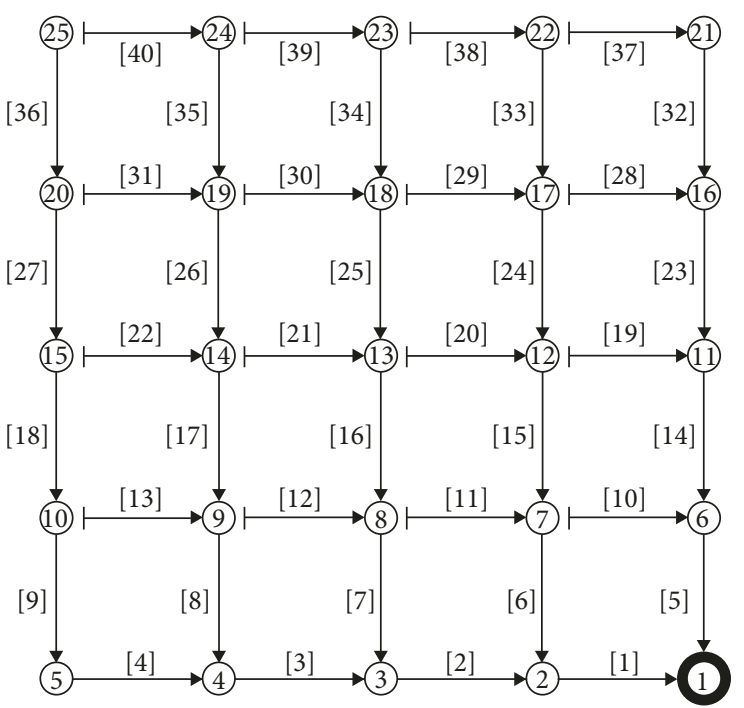

(b)

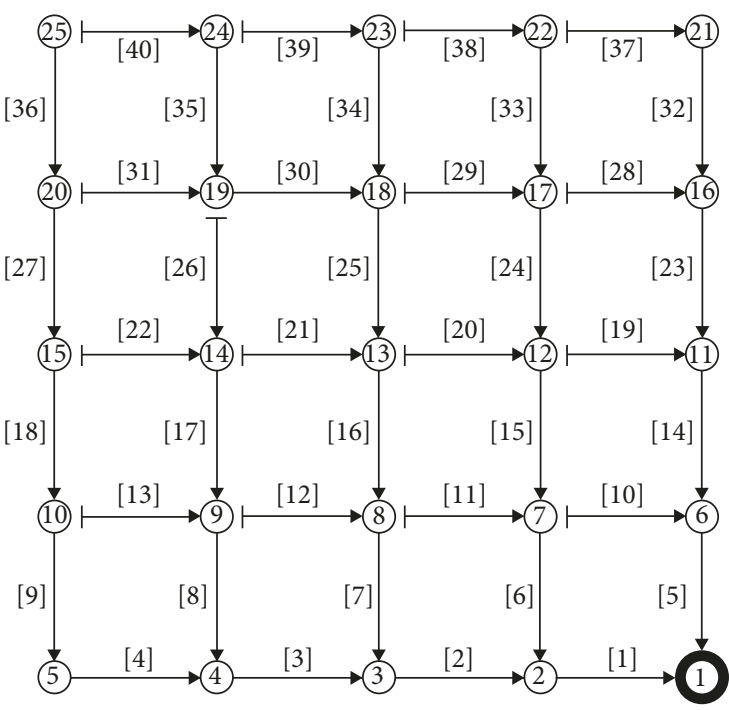

(d)

Figure 12: The four different consequence graphs.

proposed in the previous studies. This is shown in two case studies undertaken. In the first case study, the feasible layout is obtained by using Dijkstra's shortest-path algorithm in the third step of the proposed method. The results have been found to be consistent with the shortest-path solution available for the same sewer network in literature. In the second case study, the method was developed for the studies that require more than one layout.

Based on the results of the case studies undertaken, the graph theory-based method proposed in this study has proved to be successful to find the global optimum in the sewer system optimization process. Moreover, the method proposed obtains a feasible sewer system layout that contains all sewer links and satisfies all restrictions. The hydraulic design that will be performed based on the optimal layout developed using the graph theory-based methodology will no doubt be more effective and economical. This study is also expected to stimulate the use of graph theory on similar studies.

\section{Data Availability}

The data used to support the findings of this study are included within the article.

\section{Disclosure}

Part of this study is presented in the International Symposium on Urban Water and Wastewater Management (UKSAY)-2018 and published in the proceeding.

\section{Conflicts of Interest}

The authors declare that they have no conflicts of interest. 


\section{References}

[1] J. Ellis, B. Chocat, S. Fujita, J. Marsalek, and W. Rauch, "Urban drainage: a multilingual glossary," Water Intelligence Online, vol. 5, p. 9781780402536, 2015.

[2] A. Haghighi, "Loop-by-Loop cutting algorithm to generate layouts for urban drainage systems," Journal of Water Resources Planning and Management, vol. 139, no. 6, pp. 693703, 2013.

[3] A. Ahmadi, M. A. Zolfagharipoor, and M. Nafisi, "Development of a hybrid algorithm for the optimal design of sewer networks," Journal of Water Resources Planning and Management, vol. 144, no. 8, article 04018045, 2018.

[4] R. Moeini and M. H. Afshar, "Arc based ant colony optimization algorithm for optimal design of gravitational sewer networks," Ain Shams Engineering Journal, vol. 8, no. 2, pp. 207-223, 2017.

[5] A. F. Diogo, G. A. Walters, E. R. d. Sousa, and V. M. Graveto, "Three-dimensional optimization of urban drainage systems," Computer-Aided Civil and Infrastructure Engineering, vol. 15, no. 6, pp. 409-425, 2000.

[6] A. F. Diogo and V. M. Graveto, "Optimal layout of sewer systems: a deterministic versus a stochastic model," Journal of Hydraulic Engineering, vol. 132, no. 9, pp. 927-943, 2006.

[7] G. Li and R. G. S. Matthew, "New approach for optimization of urban drainage systems," Journal of Environmental Engineering, vol. 116, no. 5, pp. 927-944, 1990.

[8] L. W. Mays and H. G. Wenzel, "Optimal design of multilevel branching sewer systems," Water Resources Research, vol. 12, no. 5, pp. 913-917, 1976.

[9] L. W. Mays and B. C. Yen, "Optimal cost design of branched sewer systems," Water Resources Research, vol. 12, no. 1, pp. 37-47, 1975.

[10] M. H. Afshar, A. Afshar, M. A. Mariño, and A. A. S. Darbandi, "Hydrograph-based storm sewer design optimization by genetic algorithm," Canadian Journal of Civil Engineering, vol. 33, no. 3, pp. 319-325, 2006.

[11] M. H. Afshar, "A parameter free continuous ant colony optimization algorithm for the optimal design of storm sewer networks: constrained and unconstrained approach," $A d$ vances in Engineering Software, vol. 41, no. 2, pp. 188-195, 2010.

[12] T. Cetin and M. A. Yurdusev, "Genetic algorithm for networks with dynamic mutation rate," Gradevinar, vol. 69, no. 12, pp. 1101-1109, 2018.

[13] L. Y. Liang, R. G. Thompson, and D. M. Young, "Optimising the design of sewer networks using genetic algorithms and tabu search," Engineering, Construction and Architectural Management, vol. 11, no. 2, pp. 101-112, 2004.

[14] T.-C. Pan and J.-J. Kao, "Ga-qp model to optimize sewer system design," Journal of Environmental Engineering, vol. 135, no. 1, pp. 17-24, 2009.

[15] A. Haghighi, "Intelligent Optimization of Wastewater Collection Networks," in Intelligence Systems in Environmental Management: Theory and Applications, C. Kahraman and I. U. Sari, Eds., pp. 41-65, Springer International Publishing, Cham, Switzerland, 2017.

[16] P. K. Navin, Y. P. Mathur, and D. Kumar, "Layout optimization of sewer network using minimum cumulative flow in the sewer network," in Advances in Waste Management, pp. 333-343, Springer, Singapore, 2019.

[17] S. Tekeli and H. Belkaya, "Computerized layout generation for sanitary sewers," Journal of Water Resources Planning and Management, vol. 112, no. 4, pp. 500-515, 1986.
[18] G. A. Walters and T. Lohbeck, "Optimal layout of tree networks using genetic algorithms," Engineering Optimization, vol. 22, no. 1, pp. 27-48, 1993.

[19] R. Moeini and M. H. Afshar, "Layout and size optimization of sanitary sewer network using intelligent ants," in Advances in Engineering Software, vol. 51, pp. 49-62, Elsevier, Amsterdam, Netherlands, 2012.

[20] R. Moeini and M. H. Afshar, "Constrained ant colony optimisation algorithm for the layout and size optimisation of sanitary sewer networks," Urban Water Journal, vol. 10, no. 3, pp. 154-173, 2013.

[21] J. F. Barlow, A. Wilson, I. Heggie, V. Lane, A. Templeman, and H. Harriman, "Cost optimization of pipe sewerage systems," ICE Proceedings, vol. 53, no. 3, pp. 57-64, 1972.

[22] R. Moeini and M. H. Afshar, "Sewer Network Design Optimization Problem Using Ant Colony Optimization Algorithm and Tree Growing Algorithm," in EVOLVE-A bridge between probability, set oriented numerics, and evolutionary computation IV, Advances in Intelligent Systems and Computing, M. Emmerich, Ed., vol. 227, pp. 91-105, Springer, Heidelberg, 2013.

[23] A. Haghighi and A. E. Bakhshipour, "Deterministic integrated optimization model for sewage collection networks using tabu search," Journal of Water Resources Planning and Management, vol. 141, no. 1, article 04014045, 2015.

[24] P. K. Navin and Y. P. Mathur, "Layout and component size optimization of sewer network using spanning tree and modified PSO algorithm," Water Resources Management, vol. 30, no. 10, pp. 3627-3643, 2016.

[25] G. A. Walters and D. K. Smith, "Evolutionary design algorithm for optimal layout of tree networks," Engineering Optimization, vol. 24, no. 4, pp. 261-281, 1995.

[26] M. E. Turan, T. Bacak-Turan, T. Cetin, and E. Aslan, "Use of graph theory in sewer system optimization," in Proceedings of the International Symposium on Urban Water and Wastewater Management, Denizli, Turkey, 2018.

[27] J. L. Gross and J. Yellen, Graph Theory and Applications, Hermes, Paris, France, 2006.

[28] R. A. Brualdi and H. J. Ryser, Combinatorial Matrix Theory, Cambridge University Press, Cambridge, UK, 1991.

[29] E. W. Dijkstra, "A note on two problems in connexion with graphs," Numerische Mathematik, vol. 1, no. 1, pp. 269-271, 1959. 


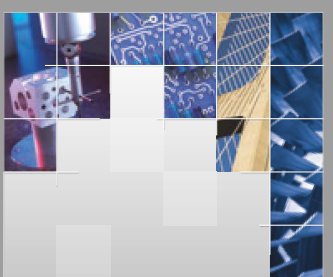

\section{Enfincering}
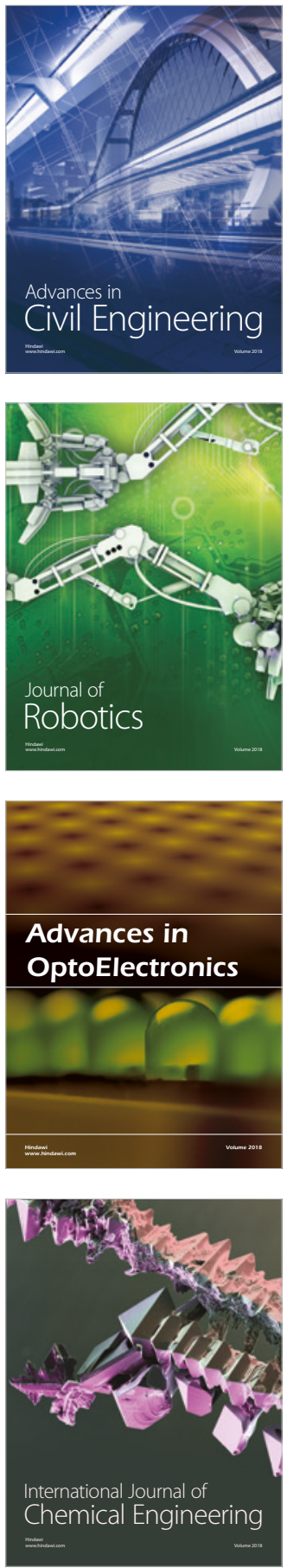

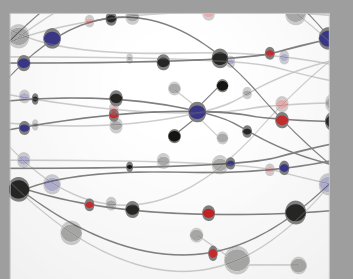

\section{Rotating \\ Machinery}

The Scientific World Journal

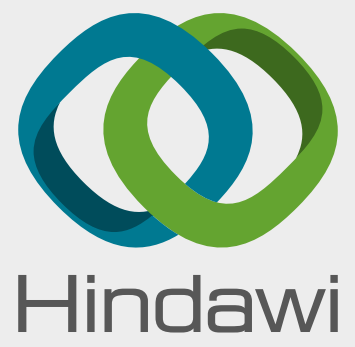

Submit your manuscripts at

www.hindawi.com
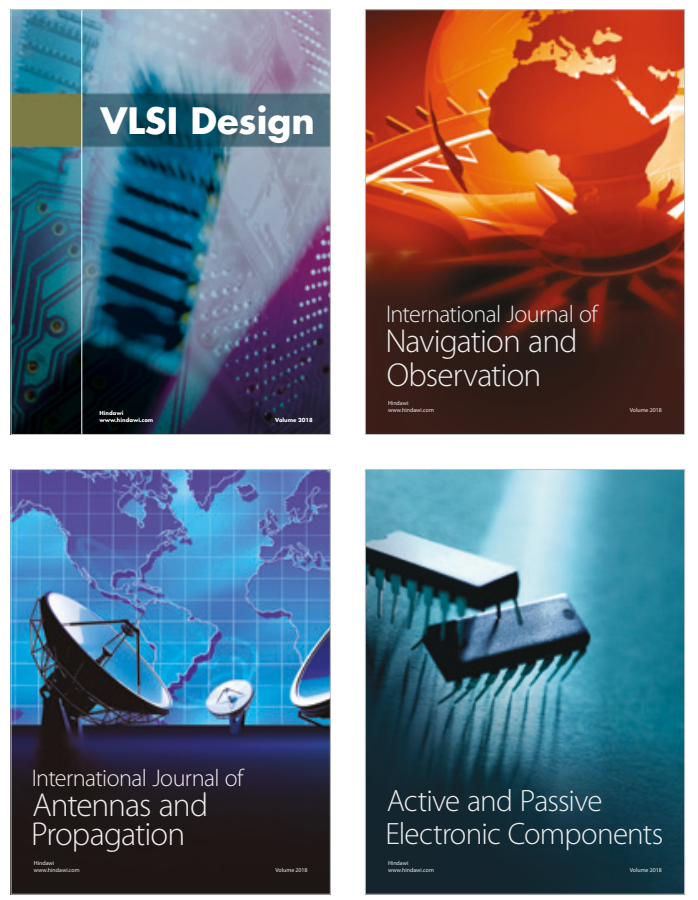
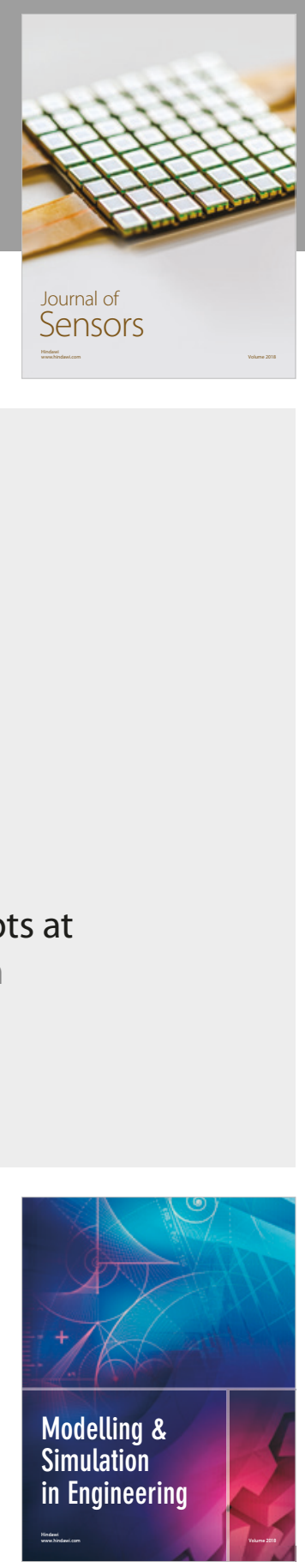

\section{Advances \\ Multimedia}
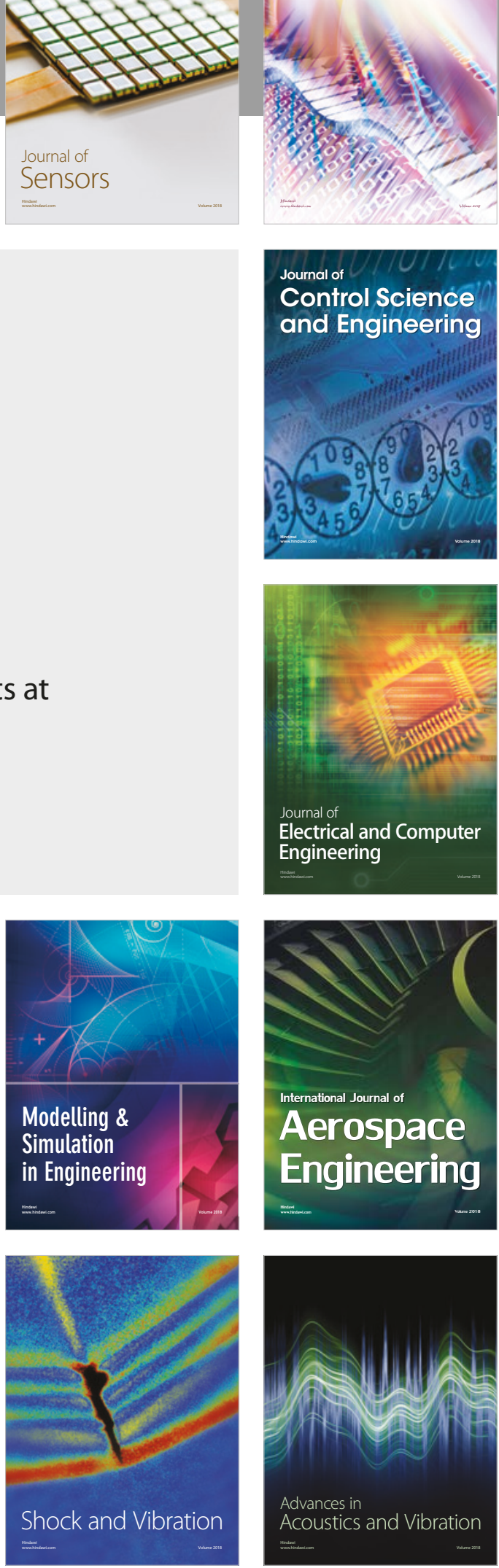